\title{
Dynamics of Heading and Choice-Related Signals in the Parieto-Insular Vestibular Cortex of Macaque Monkeys
}

\author{
Aihua Chen, ${ }^{1}$ Fu Zeng, ${ }^{1}{ }^{\circledR}$ Gregory C. DeAngelis, ${ }^{2}$ and Dora E. Angelaki ${ }^{3,4}$ \\ ${ }^{1}$ Key Laboratory of Brain Functional Genomics, East China Normal University, Shanghai 200062, China, ${ }^{2}$ Department of Brain and Cognitive \\ Sciences, Center for Visual Science, University of Rochester, Rochester, New York 14627, ${ }^{3}$ Department of Neuroscience, Baylor College of Medicine, \\ Houston, Texas 77030, and ${ }^{4}$ Center for Neural Science and Tandon School of Engineering, New York University, New York, New York 10003
}

Perceptual decision-making is increasingly being understood to involve an interaction between bottom-up sensory-driven signals and top-down choice-driven signals, but how these signals interact to mediate perception is not well understood. The parieto-insular vestibular cortex (PIVC) is an area with prominent vestibular responsiveness, and previous work has shown that inactivating PIVC impairs vestibular heading judgments. To investigate the nature of PIVC's contribution to heading perception, we recorded extracellularly from PIVC neurons in two male rhesus macaques during a heading discrimination task, and compared findings with data from previous studies of dorsal medial superior temporal (MSTd) and ventral intraparietal (VIP) areas using identical stimuli. By computing partial correlations between neural responses, heading, and choice, we find that PIVC activity reflects a dynamically changing combination of sensory and choice signals. In addition, the sensory and choice signals are more balanced in PIVC, in contrast to the sensory dominance in MSTd and choice dominance in VIP. Interestingly, heading and choice signals in PIVC are negatively correlated during the middle portion of the stimulus epoch, reflecting a mismatch in the polarity of heading and choice signals. We anticipate that these results will help unravel the mechanisms of interaction between bottom-up sensory signals and top-down choice signals in perceptual decision-making, leading to more comprehensive models of self-motion perception.

Key words: bottom-up; choice; partial correlation; PIVC; sensory; top-down

Significance Statement

Vestibular information is important for our perception of self-motion, and various cortical regions in primates show vestibular heading selectivity. Inactivation of the macaque vestibular cortex substantially impairs the precision of vestibular heading discrimination, more so than inactivation of other multisensory areas. Here, we record for the first time from the vestibular cortex while monkeys perform a forced-choice heading discrimination task, and we compare results with data collected previously from other multisensory cortical areas. We find that vestibular cortex activity reflects a dynamically changing combination of sensory and choice signals, with both similarities and notable differences with other multisensory areas.

\section{Introduction}

Vestibular information is important for spatial orientation, postural control, balance, and navigation (Stackman et al., 2002; McNaughton et al., 2006; Yoder and Taube, 2014). Signals from the otolith organs, in particular, provide a powerful cue for

Received Aug. 29, 2020; revised Jan. 20, 2021; accepted Feb. 17, 2021.

Author contributions: G.C.D. and D.E.A. designed research; A.C. performed research; A.C. contributed unpublished reagents/analytic tools; A.C. and F.Z. analyzed data; A.C., G.C.D., and D.E.A. wrote the paper.

D.E.A. was supported by the National Institutes of Health grant Grant R01 DC014678. G.C.D. was supported by the National Institutes of Health grant Grant R01 EY016178. We thank A. Turner and E. White for monkey care and training as well as Jose A. Fernandez-Leon for work on an early version of the partial correlation analysis and manuscript preparation.

The authors declare no competing financial interests.

Correspondence should be addressed to Dora E. Angelaki at da93@nyu.edu.

https://doi.org/10.1523/JNEUROSCI.2275-20.2021

Copyright $\odot 2021$ the authors detecting linear acceleration and perceiving heading (Telford et al., 1995; Ohmi, 1996; Harris et al., 2000; Gu et al., 2007). Several cortical areas have been observed to respond to vestibular signals with similar heading tuning ( $\mathrm{Gu}$ et al., 2006; Chen et al., 2011a,b), and task-related activity during vestibular heading discrimination has been reported in the dorsal medial superior temporal area (MSTd; Gu et al., 2007) and the ventral intraparietal area (VIP; Chen et al., 2013) of macaque monkeys. However, despite the similarity of vestibular heading responses across areas, inactivation of these areas had different effects on vestibular heading perception. MSTd inactivation had significant, but relatively weak, effects on vestibular heading discrimination ( $\mathrm{Gu}$ et al., 2012), while large bilateral muscimol injections into area VIP had revealed no deficits in performance (Chen et al., 2016). In contrast, inactivation of parieto-insular vestibular cortex (PIVC) had a strong effect on vestibular heading perception (Chen et al., 
2016). To examine how PIVC contributes to vestibular heading perception, extracellular neuronal activity was recorded while monkeys performed a forced-choice heading discrimination task, and results were compared with data collected previously from areas MSTd and VIP during the same task (Gu et al., 2007, 2008; Chen et al., 2013).

Since PIVC is strongly influenced by spatial-temporal acceleration signals, as compared with MSTd and VIP (Chen et al., 2011c), we first examined whether psychophysical heading sensitivity is consistent with the sensitivity of PIVC neurons at two time windows around peak and trough acceleration. Using signal detection theory to convert neural activity into neurometric functions that can be compared with psychometric functions, we found that almost all PIVC neurons were less sensitive than the monkey to small changes in heading in both time windows. However, the average sensitivity of PIVC neurons was comparable to that of neurons in MSTd or VIP. These observations suggest that the more acceleration-dominant signals in PIVC do not afford substantially greater sensitivity, and that the stronger effects of reversible inactivation of PIVC, relative to MSTd and VIP, are not simply related to a difference in sensitivity between areas.

Since tuning functions measured while animals perform perceptual decision-making tasks could be confounded by choicedriven signals arising from a top-down source (Cumming and Nienborg, 2016; Goris et al., 2017; Zaidel et al., 2017), studying the neural basis of perception also benefits from examining the dynamic interaction between stimulus-related and choice-related signals. We performed a partial correlation analysis (Zaidel et al., 2017) to examine how neural responses depend on both stimulus (heading) and choice, as well as how these relationships change as a function of time. We found that the responses of most PIVC neurons reflect a roughly balanced combination of stimulus and choice signals. By comparison, responses in MSTd are dominated by stimulus heading and VIP activity is dominated by choice signals throughout the stimulus period. Interestingly, heading and choice signals in PIVC are negatively correlated during the middle portion of the stimulus epoch, indicating that choice signals in PIVC often have an opposing influence relative to sensory signals. These results demonstrate that PIVC not only encodes the stimulus (e.g., acceleration signals) but also choice signals, and the dynamic interaction between these two balanced signals might play an important role during heading perception.

\section{Materials and Methods}

\section{Surgical procedures}

Four male rhesus monkeys (Macaca mulatta), weighing from 6 to $10 \mathrm{~kg}$, contributed data to the current study: two of the animals (Monkeys A and C) were used as part of a previous study of MSTd (Gu et al., 2007), two of the animals (Monkeys $\mathrm{U}$ and $\mathrm{C}$ ) were part of a previous study of VIP (Chen et al., 2013), and three of the animals (Monkeys U, C, and J) contributed to the PIVC recordings described here. Data from PIVC were collected between June 2007 and July 2008; experiments in all three areas were conducted using the same experimental apparatus, as described below.

The animals were chronically implanted with a circular molded, lightweight Delrin ring for head restraint, a neural recording grid for placing electrodes, and a scleral search coil for measuring eye movements. Animals were trained using standard operant conditioning to fixate visual targets for fluid reward, and to report their direction of heading as described below. All animal surgeries and experimental procedures were approved by the Institutional Animal Care and Use Committee at Washington University in St. Louis and were in accordance with National Institutes of Health guidelines.

\section{Magnetic resonance imaging (MRI) acquisition and} electrophysiology

Areas PIVC, VIP, and MSTd were identified based on patterns of gray and white matter transitions along electrode penetrations with respect to MRI scans, recording depths, and physiological response properties, as detailed in previous studies (Gu et al., 2006; Takahashi et al., 2007; Chen et al., 2010, 2011a). All PIVC neurons were located in the upper bank and tip of the lateral sulcus. MSTd and VIP data presented here are from previous studies (Gu et al., 2007 for MSTd; Chen et al., 2013 for VIP).

Standard methods for single unit extracellular recording were used. Briefly, a tungsten microelectrode (Frederick Haer Company; tip diameter $3 \mu \mathrm{m}$, impedance $1-2 \mathrm{M} \Omega$ at $1 \mathrm{kHz}$ ) was advanced into the cortex through a transdural guide tube using a micromanipulator (Frederick Haer Company) mounted on top of the head-restraint ring. Single neurons were isolated using a conventional amplifier with a bandpass eightpole filter $(400-5000 \mathrm{~Hz})$ and a dual voltage-time window discriminator (Bak Electronics). The times of action potentials and all behavioral events were recorded with 1-ms resolution. Raw neural signals were also digitized at $25 \mathrm{kHz}$ and stored for off-line spike sorting and additional analyses. Eye movement traces were low-pass filtered and sampled at $250 \mathrm{~Hz}$.

\section{Motion platform and heading stimuli}

Vestibular stimuli were delivered by a six-degree of freedom motion platform (MOOG 6DOF2000E). The trajectory of inertial motion was controlled in real time at $60 \mathrm{~Hz}$ over an Ethernet interface. A three chip DLP projector (Christie Digital Mirage 2000) was mounted on top of the platform and rear-projected images onto a $60 \times 60 \mathrm{~cm}$ tangent screen attached to the front of the field coil frame. The display was viewed from a distance of $30 \mathrm{~cm}$, thus subtending $90 \times 90^{\circ}$ of visual angle. The sides of the coil frame were covered with a black enclosure. Visual stimuli were generated by an OpenGL accelerator board (nVidia Quadro FX $3000 \mathrm{G}$ ). The display had a pixel resolution of $1280 \times 1240$, 32-bit color depth, and was updated at the same rate as the movement trajectory $(60 \mathrm{~Hz})$.

\section{Heading discrimination task}

Monkeys were trained using standard operant conditioning techniques to report their direction of translation during a two-alternative, forcedchoice heading discrimination task (Fig. $1 A$ ). In each trial, the monkey was moved along one of several directions in the horizontal plane (leftward or rightward relative to straight ahead; Fig. $1 A$ ) while maintaining fixation on a central target (Fig. 1B). The fixation point remained fixed relative to the head during motion. Trials were aborted if the monkey's conjugate eye position deviated from a $2 \times 2^{\circ}$ window around the fixation point. The motion trajectory was $2 \mathrm{~s}$ in duration and followed a Gaussian velocity profile (Fig. 1C, dashed curve) with a corresponding biphasic linear acceleration profile measured during vestibular movement (Fig. 1C, solid curve). The total displacement along the motion trajectory was $30 \mathrm{~cm}$, with a peak acceleration of $\pm 0.1 \mathrm{G}\left( \pm 0.98 \mathrm{~m} / \mathrm{s}^{2}\right)$ and a peak velocity of $45 \mathrm{~cm} / \mathrm{s}$.

To report their heading, animals were trained to make a saccade to one of two targets that appeared $5^{\circ}$ left and right of the fixation point after termination of the motion stimulus (Fig. $1 B$ ). The saccade had to be made within 1s of the appearance of the two targets, and the saccade endpoint had to remain within $3^{\circ}$ of the target for at least $150 \mathrm{~ms}$. Correct responses were rewarded with a drop of water or juice. For the ambiguous heading condition $\left(0^{\circ}\right.$, straight ahead), rewards were delivered randomly on half of the trials.

Across trials, heading was varied in fine steps around straight ahead. The range of headings was chosen based on extensive psychophysical testing using a staircase paradigm. Nine logarithmically spaced heading angles, including an ambiguous straight-ahead direction $\left( \pm 9^{\circ}, \pm 3.5^{\circ}\right.$, $\pm 1.3^{\circ}, \pm 0.5^{\circ}$, and $0^{\circ}$ ) were used for all experiments except for the MSTd recordings from Monkey $\mathrm{C}$, in which case the following values were used: $\pm 16^{\circ}, \pm 6.4^{\circ}, \pm 2.5^{\circ}, \pm 1^{\circ}$, and $0^{\circ}$. These headings were chosen to obtain near-maximal psychophysical performance while allowing neural sensitivity to be estimated reliably for most neurons. The average psychophysical thresholds were $2.75 \pm 0.15 \mathrm{SE}$ for Monkey U, $2.87 \pm 0.21$ 
SE for Monkey J, 1.22 \pm 0.02 SE for Monkey $\mathrm{A}$, and $2.32 \pm 0.09 \mathrm{SE}$ for Monkey C.

\section{Experimental protocols}

Once a single neuron was isolated, only cells with clear, audible response modulation to sinusoidal translation or rotation were measured vestibular heading tuning in threedimensions. Stimuli consisted of $2 \mathrm{~s}, 13 \mathrm{~cm}$, $0.1 \mathrm{G}$ displacements along each of 26 directions spanning 3D space (Chen et al., 2010) and were presented four to five times each during passive fixation of a head-centered target. If good cell isolation was maintained after the $3 \mathrm{D}$ tuning protocol, we further tested the neuron with the vestibular heading discrimination task. Because the MSTd and VIP data reported here for comparison were recorded as part of different studies, somewhat different criteria were used to search for and select neurons. In MSTd, only neurons with significant vestibular tuning in the horizontal plane were tested during the heading discrimination task (Gu et al., 2007); in contrast, VIP neurons with either significant vestibular or visual tuning were further tested with heading discrimination task (Chen et al., 2013). Thus, to allow a fair comparison among areas, we only include cells here with significant temporal response modulation and significant directional tuning in response to vestibular translation in the horizontal plane (criteria described below). Accordingly, we selected subsamples of the MSTd and VIP populations that met the same criteria used to select PIVC neurons.

\section{Data analysis}

Spatiotemporal analysis of PIVC responses PSTHs were constructed for each heading using 25-ms time bins, and were smoothed with a 400-ms boxcar filter. The temporal modulation along each stimulus direction was considered significant when the spike count distribution from the time bin containing the maximum and/or minimum response differed significantly from the baseline response distribution from -100 to $300 \mathrm{~ms}$ poststimulus onset (Wilcoxon signed-rank test, $p<0.01$ ). For neurons with a minimum of two adjacent $\left(\leq 45^{\circ}\right)$ stimulus directions in the horizontal plane having significant temporal modulation, we calculated the maximum (for excita-

tory cells that show facilitation relative to baseline response) or minimum (for inhibitory cells that show suppression relative to baseline) response across stimulus directions (Chen et al., 2010). This was performed for each 25-ms time bin between $0.5-2 \mathrm{~s}$ after motion onset. An ANOVA was performed to assess the statistical significance of directional selectivity for each time bin. This analysis determined the significance of direction tuning in the horizontal plane as a function of time and assessed whether there are multiple periods in which a neuron shows distinct temporal peaks of directional tuning (Chen et al., 2010). "Peak times" were then defined as the times of local maxima (for excitatory cells) or minima (for inhibitory cells) at which distinct epochs of directional tuning were observed. Cells with no peak times (i.e., no significant directional tuning at any time during the response window) were excluded from further analysis. Thus, cells included in the present study, from all three brain areas, were required to have significant temporal response modulation and significant directional tuning. Excitatory cells are much more common than inhibitory cells (Chen et al., 2010), and all PIVC neurons tested during the heading discrimination task were excitatory cells.

\section{ROC analysis}

Behavioral performance was quantified by plotting the proportion of "rightward" choices as a function of heading (angular orientation of the motion direction from straight ahead) and psychometric functions were fit with a cumulative Gaussian function:

$$
P(h)=\frac{1}{\sigma \sqrt{2 \pi}} \int_{-\infty}^{h} e^{\frac{-(x-\mu)^{2}}{2 \sigma^{2}}} d x .
$$

Psychophysical threshold was defined as the standard deviation of the Gaussian fit, $\sigma$, which corresponds to $84 \%$ correct performance (assuming no bias). Neural sensitivity was quantified by applying ROC analysis to distributions of spike counts for pairs of leftward versus 
rightward headings (e.g., $-9^{\circ}$ vs $+9^{\circ}$ ). The resulting neurometric functions were then fit with a cumulative Gaussian function to estimate neuronal thresholds (for details, see Gu et al., 2007; Chen et al., 2013).

\section{Partial correlation analysis}

To disassociate the contributions of vestibular heading stimuli and choice to neural responses (spike counts), we computed Pearson partial correlations between each of these variables (Prokhorov, 2001; Zaidel et al., 2017). This produced a heading partial correlation, $r_{\mathrm{FH} \mid \mathrm{C}}$, that captured the linear relationship between firing rate $(F)$ and heading $(H)$ given the monkey's choice $(C)$, as well as a choice partial correlation, $\mathrm{r}_{\mathrm{FC} \mid \mathrm{H}}$, that captured the relationship between firing rate and choice given the stimulus heading.

Partial correlations were calculated over the entire 2-s stimulus duration as well as in non-overlapping $100-\mathrm{ms}$ time windows starting from stimulus onset. Positive heading partial correlations indicate that firing rates were greater for rightward than leftward headings. Likewise, positive choice partial correlations indicate that firing rates were greater for choices made to the right than choices made to the left.

\section{Autoregressive, integrated moving average (ARIMA) model}

The time course of partial correlations was analyzed by fitting an appropriate statistical model, ARIMA (Newbold, 1983), which is descriptive and captures a range of different temporal structure in time series data. The model has an autoregressive (AR) part, meaning that it makes use of the dependent relationship between an observation and some number of previous (lagged) observations. The model has an integrative (I) component, in that it uses differencing of raw observations (e.g., subtracting an observation from an observation at the previous time step) to make the time series stationary. And the model involves a moving average (MA), in which the dependency between an observation and a residual error from a MA is applied to lagged observations.

The underlying time series has the following form in the ARIMA model:

$$
y_{t}^{\prime}=c+\Phi_{1} y_{t-1}^{\prime}+\ldots+\Phi_{p} y_{p-1}^{\prime}+\theta_{1} e_{t-1}+\ldots+\theta_{q} e_{q-1}+e_{t}
$$

where $c$ is a constant, $y_{t}^{\prime}$ is the differenced time series of observations, $\mathrm{e}_{\mathrm{t}}$ is the random error or white noise at a time $t, \Phi_{1}, \Phi_{2}, \ldots, \Phi_{\mathrm{p}}$ are the coefficients of the AR part of the $\mathrm{p}^{\text {th }}$ order, and $\theta_{1}, \theta_{2}, \ldots, \theta_{\mathrm{q}}$ are the coefficients of the moving-average part of the qth order, respectively.

A standard form of the model is defined as ARIMA (p,d,q), where $p$ is the order of the AR term, $d$ is the degree of first differencing involved to make the time series stationary, and $\mathrm{q}$ is the order of the MA. A parameter value of 0 indicates that element of the model is not used, such that the ARIMA model can simplify down to an AR, I, or MA model. As an example, $\operatorname{ARIMA}(1,0,12)$ means that the model describes some response variable $(\mathrm{Y})$ by combing a first order Auto-Regressive model and a 12th order MA model.

To find the best values of $\mathrm{p}, \mathrm{d}$ and $\mathrm{q}$, we first check whether the time series is stationary (e.g., constant mean and variance) with the augmented Dickey-Fuller test (ADF test in MATLAB), since it is difficult to model a series that changes over time. The null hypothesis of the ADF test is that the time series is non-stationary. Thus, if the $p$ value of the test is less than the significance level (0.05), one rejects the null hypothesis and infers that the time series is indeed stationary. Differencing is necessary only if the series is non-stationary, and one seeks the lowest order of differencing required to get a near stationary series. By making the data stationary, one can apply regression techniques to the time dependent variable.

We used both Akaike's information criterion (AIC) and Schwartz Bayesian information criterion (BIC) to select the degrees $\mathrm{p}$ and $\mathrm{q}$ of the model (aicbic function in MATLAB). We estimated several models with different $\mathrm{p}$ and $\mathrm{q}$ values within a certain range $(0 \sim 5$ in our study), then investigated the models with the lowest sum of AIC and BIC values, as AIC was useful in selecting predictors for regression and BIC generally penalized free parameters more strongly. We did not use the traditional method of autocorrelation functions (ACFs) and partial ACFs (PACF) to estimate the values of $\mathrm{p}$ and $\mathrm{q}$, since the number of datapoints for our time course of partial correlation was relatively small.

Finally, we plot the residual distribution, the ACF and PACF, to see whether our model is good. If the residual is normally distributed, and there are no significant terms in the ACF and PACF, we considered the model was performing well.

To compare two time series datasets, we first fit each time series separately with the ARIMA model. Then we estimate the two time series datasets jointly by putting the second series behind the first, and perform a "chow test" (in MATLAB) to examine the hypothesis of a common set of parameters. The basic idea here is to test whether the same set of parameters (which make up the ARIMA model) can describe both time series. If the fits of two time series are superior to the joint fit with a common set of parameters (chow test), then we classify the two time series as significantly different.

\section{Results}

\section{Psychophysical performance}

To assess perception of self-motion on the basis of vestibular cues in PIVC, three animals (Monkeys U, J, and C) were trained to report their direction of translation in a two-alternative, forced-choice heading discrimination task. On each trial, the monkey was translated along one of nine directions in the horizontal plane while fixation was maintained on a central target (Fig. 1A). At the end of each trial, the animal reported its movement as either rightward or leftward relative to straight ahead by making a saccade to one of two targets presented at the end of the movement (Fig. 1B). The inertial motion stimulus had a Gaussian velocity profile with a peak acceleration of $1 \mathrm{~m} / \mathrm{s}^{2}$ (Fig. $1 C)$. Average psychometric functions are shown for two monkeys (U: $N=75$ recording sessions; J: $N=29$ recording sessions) in Figure $1 D$, black symbols. Here, we did not include the behavioral data from Monkey $\mathrm{C}$ since only four recording sessions were performed in this monkey. The proportion of rightward decisions is plotted against heading and the data were fit with a cumulative Gaussian function (red curves). Psychophysical threshold is defined as the standard deviation of the cumulative Gaussian that best fits the data, which corresponds to $84 \%$ correct performance in the absence of bias. Both animals discriminated small changes in heading based on inertial motion cues, with average psychophysical thresholds of $2.57 \pm 0.18^{\circ} \mathrm{SE}$ for Monkey $\mathrm{U}$ and $2.87 \pm 0.21^{\circ} \mathrm{SE}$ for Monkey J, respectively. These heading thresholds are comparable to those found for both macaques and humans in previous studies (Gu et al., 2007, 2008; Fetsch et al., 2010; Chen et al., 2013).

\section{PIVC responses to translation motion}

We previously presented a thorough description of the responses of PIVC neurons to translation movements (Chen et al., 2010). Following our previous work (Chen et al., 2010), neurons showing a single epoch of directional selectivity in which the heading preference remains constant over time are referred to as "singlepeaked" (SP) cells (Fig. 2A for PSTHs in spherical coordinates; Fig. $2 C$ for $3 \mathrm{D}$ heading tuning profile at peak time). In contrast, "double-peaked" (DP) neurons that follow the linear acceleration profile of the motion trajectory exhibit two peaks of directional selectivity separated in time, with opposite preferences (Fig. $2 B$ for PSTHs in spherical coordinates; Fig. $2 D, E$ for $3 \mathrm{D}$ heading tuning at early and late peak times, respectively).

In the current study, we first determined the spatiotemporal tuning of each cell within the horizontal plane before we recorded from neurons during the heading discrimination task. Thus, we also classified neurons as SP and DP in the horizontal plane. An example SP cell is shown in Figure 3A. The response 
A

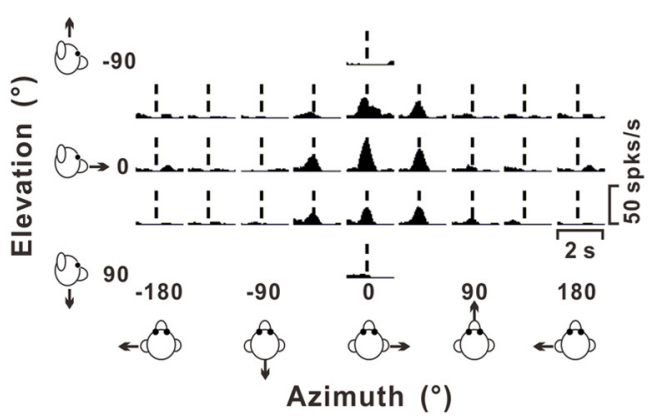

B

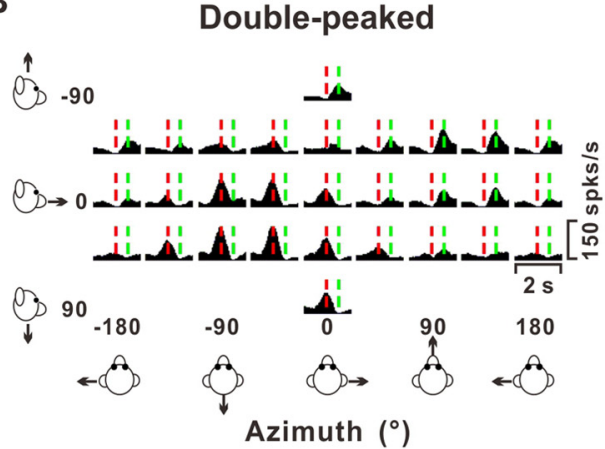

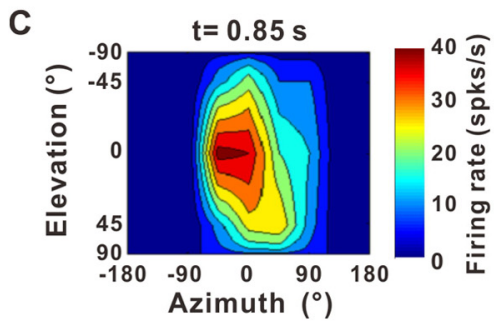
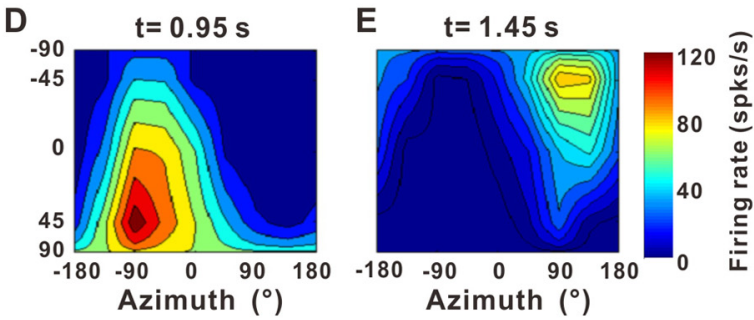

Figure 2. Example responses from SP and DP cells during measurements of 3D heading tuning. $\boldsymbol{A}, \boldsymbol{B}$, Response PSTHs for example neurons having SP $(\boldsymbol{A})$ and DP $(\boldsymbol{B})$ dynamics. Each PSTH shows the mean response to one stimulus direction defined in spherical coordinates by its azimuth (varying along the abscissa) and elevation (varying along the ordinate) angles. Vertical dashed black lines $(t=0.85 \mathrm{~s})$ in panel $\boldsymbol{A}$ indicate the time at which the maximum response across directions occurred (peak time). Vertical dashed red $(t=0.95 \mathrm{~s})$ and green $(t=1.45 \mathrm{~s})$ lines in panel $\boldsymbol{B}$ illustrate the two peak times for the DP cell. PSTHs were computed with sequential 25 -ms bins and then smoothed with a 400-ms sliding window. $C$, Color contour map showing the 3D heading tuning profile (Lambert cylindrical projection) at peak time for the SP cell of panel $A$. This SP cell was significantly tuned for heading (ANOVA, $p=3.3 \times 10^{-9}$ ), with a preferred direction (computed as the vector sum of response) at azimuth $=11^{\circ}$ and elevation $=3^{\circ}$, corresponding to a rightward and slightly downward movement. $\boldsymbol{D}, \boldsymbol{E}$, Color contour maps showing $3 \mathrm{D}$ heading tuning profiles at the two peak times for the DP cell of panel $\boldsymbol{B}$. The direction tuning at the first peak time had a heading preference at [azimuth, elevation] $=\left[-60^{\circ}\right.$, $\left.36^{\circ}\right](\boldsymbol{D})$, whereas the later peak of tuning was centered at [azimuth, elevation] $=\left[112^{\circ},-40^{\circ}\right](\boldsymbol{E})$. The difference between the two direction preferences for this neuron was $172^{\circ}$.

of this example neuron peaks at $0.94 \mathrm{~s}$ (vertical dashed black lines). An example of a DP neuron is shown in Figure 3B, with an early peak time ( $t=0.72 \mathrm{~s}$, vertical dashed red lines) for some directions that roughly corresponds to the time of peak acceleration $(t=0.82 \mathrm{~s})$ and a late peak for other directions $(t=1.18 \mathrm{~s}$, vertical dashed green lines) that roughly corresponds to the peak deceleration time $(t=1.18 \mathrm{~s})$. As shown in Figure $3 C, D$ for these two example neurons, we computed tuning curves in the horizontal plane, with mean firing rates calculated at the peak times. For the SP neuron, the direction tuning curve at the peak time $(t=0.94 \mathrm{~s})$ had a heading preference of $33^{\circ}$, which corresponds to a forward/rightward motion (Fig. 3C). For the DP neuron, the direction tuning curve at the early peak time $(t=0.72 \mathrm{~s})$ had a heading preference of $9.1^{\circ}$. At the late peak time $(t=1.18 \mathrm{~s})$, the preferred heading was $168.1^{\circ}$ (Fig. $3 D$ ). The difference between the two direction preferences for this DP neuron was $159^{\circ}$, close to the expected difference of $180^{\circ}$ corresponding to a complete reversal.

Distributions of peak times from responses tested in the horizontal plane are summarized in Figure 3E. The average peak time for SP cells $( \pm \mathrm{SE})$ is $0.88 \pm 0.04 \mathrm{~s}(N=48$; Fig. $3 E$, black $)$, which is earlier than the peak of the stimulus velocity profile, and closer to the time of peak acceleration $(0.82 \mathrm{~s})$. This might indicate that SP cells in PIVC are sensitive to velocities slower than the peak stimulus velocity, or that they have some contribution from acceleration that reduces the peak time but is not strong enough to generate a DP response profile. For DP cells, the average early peak time is $0.80 \pm 0.02 \mathrm{~s}(N=57)$, and the average late peak time is $1.28 \pm 0.03 \mathrm{~s}$ (Fig. $3 E$, red and green, respectively). These values for DP cells correspond quite closely to the times of peak acceleration $(0.82 \mathrm{~s})$ and deceleration $(1.18 \mathrm{~s})$ dictated by the motion trajectory. In addition, there are three triple peaked cells that may represent jerk (for details, see Chen et al., 2010, 2011c).

In total, 108 PIVC neurons with significant vestibular heading tuning in the horizontal plane were further tested during performance of the heading discrimination task. For comparison, we also reanalyzed data from 182 neurons from MSTd (Gu et al., 2007) and 68 neurons from VIP (Chen et al., 2013).

\section{Heading sensitivity in PIVC}

We examined the sensitivity of PIVC neurons, to assess whether they are sensitive enough to account for psychophysical performance. Figure $4 A$ shows the response PSTHs of an example PIVC neuron during the heading discrimination task. Since many PIVC cells are DP, the following analyses were all performed within two time windows (acceleration window and deceleration window) that are centered around the times of peak acceleration $(t=0.82 \mathrm{~s}$; Fig. $4 A$, vertical dashed red line $)$ and peak deceleration $(t=1.18 \mathrm{~s}$; Fig. $4 A$, vertical dashed green line), respectively. For both acceleration and deceleration windows, heading tuning curves for this example neuron were monotonic over the narrow range of headings tested (Fig. $4 B$, red and green curves, respectively), with stronger responses to rightward (positive) than leftward (negative) headings. Heading sensitivity was quantified by using ROC analysis to construct a neurometric function, and then computing the neuronal threshold from the best fitting cumulative Gaussian function (Britten et al., 1996). Neuronal thresholds were $8.6^{\circ}$ (acceleration peak time) and $6.1^{\circ}$ (deceleration peak time) for the example neuron, substantially greater than the simultaneously measured behavioral threshold $\left(1.5^{\circ}\right.$; Fig. 4C).

Summary data for the PIVC population are shown in Figure $4 D, E$. Similar to results reported previously for areas MSTd (Gu 
A
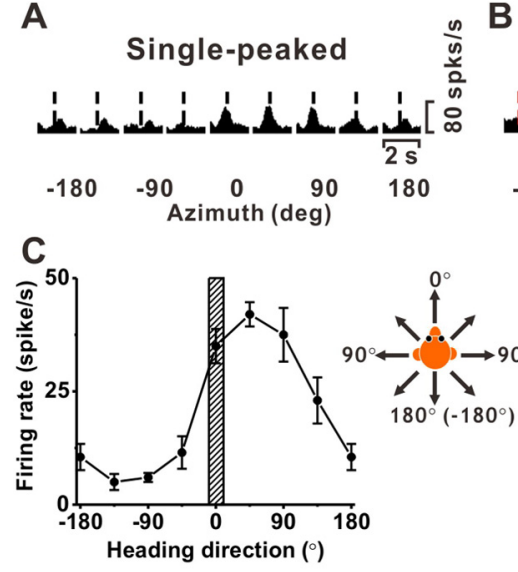

E Single-peaked
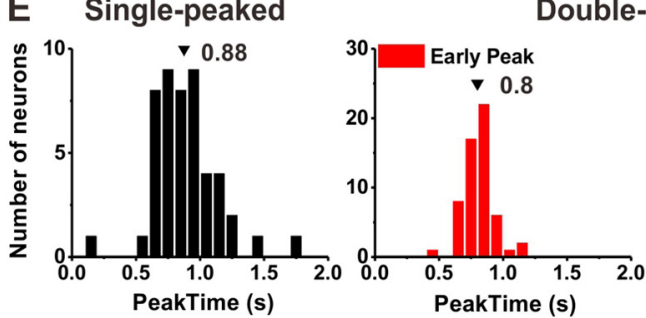

Double-peaked
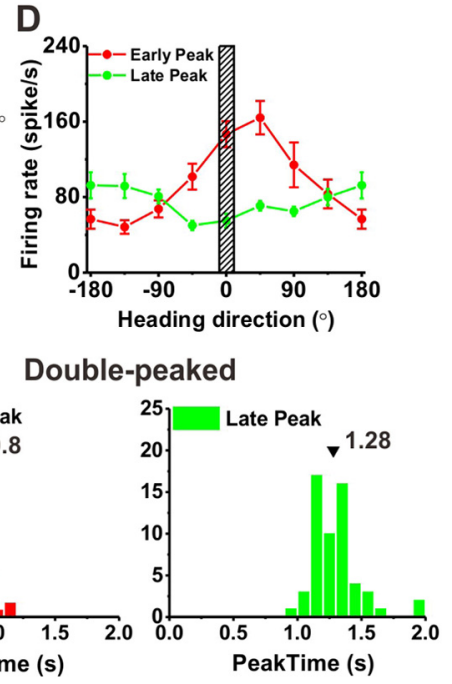

Figure 3. Vestibular tuning for heading in the horizontal plane. $\boldsymbol{A}$, Average PSTHs for eight headings in the horizontal plane for an example SP neuron. Vertical dashed black lines indicate the peak time $(t=0.93 \mathrm{~s})$. B, Average response PSTHs for an example DP neuron. Red and green dashed vertical lines indicate the early and late peak times $(t=0.68$ and $1.18 \mathrm{~s})$. C, Heading tuning curve for the SP neuron. The mean firing rate ( \pm SE) calculated at the peak time is plotted as a function of heading. The heading preference is $33^{\circ}$, which corresponds to forward/rightward motion. Striped area illustrates the narrow range of headings tested during the discrimination task. $\boldsymbol{D}$, Heading tuning curves for the DP neuron, computed at first peak time (red) and second peak time (green). $\boldsymbol{E}$, Distribution of peak times for SP cells (black), as well as the first (red) and second (green) peak times of DP cells. Arrowheads illustrate mean values.

et al., 2007) and VIP (Chen et al., 2013), the vast majority of PIVC neurons are much less sensitive than the monkey to small changes in heading. The ratio of neuronal to psychophysical thresholds from all three monkeys were $10.0 \pm 3.8$ and $9.9 \pm 4.2$ (geometric mean $\pm \mathrm{SE}$ ) for the acceleration and deceleration windows, respectively. The neuronal thresholds for Monkeys $U$ and $\mathrm{J}$ are not significantly different $(p=0.1707$ for the acceleration and $p=0.0650$ for deceleration windows, respectively, Wilcoxon rank test). Because of the small sample size $(N=4)$, we did not compare the results from Monkey $\mathrm{C}$ with those from Monkeys $\mathrm{U}$ and J. For the acceleration window, the average neuronal threshold in PIVC was 22.9 \pm 6.4 (geometric mean $\pm \mathrm{SE}$ across all animals; Fig. $4 D$ ), which is not significantly different from the average threshold for MSTd $(21.0 \pm 8.8 ; p=0.4588$, Wilcoxon rank test), but significantly greater than that for VIP $(15.2 \pm 5.3 ; p=0.0102)$. For the deceleration window (Fig. $4 E$ ), the average neuronal threshold in PIVC was $22.8 \pm 8.2$, and not significantly different from the corresponding values for MSTd $(23.1 \pm 10.0, p=0.7804$, Wilcoxon rank test) and VIP (19.8 $\pm 7.3, p=0.2154$, Wilcoxon rank test). Thus, the stronger effects of inactivating PIVC on vestibular heading perception, as compared with MSTd and VIP (Gu et al., 2012; Chen et al., 2016), do not appear to be simply explained by differences in neuronal sensitivity across areas.

We also compared the slopes of tuning curves for PIVC neurons around $0^{\circ}$ heading (forward) for the fixation and discrimination tasks. The slopes are generally consistent between these two measurement conditions and are highly correlated (acceleration window: $r=0.53, p=9.16 \times 10^{-9}$; deceleration window: $r=0.55, p=1.45 \times 10^{-9}$, Pearson's correlation). Thus, heading preferences of PIVC neurons during discrimination are largely consistent with those measured during fixation.

\section{Representation of heading and choice signals in PIVC versus MSTd and VIP} To investigate how PIVC responses are related to heading perception, we further examined the relationship between neuronal activity and perceptual decisions. Since tuning curves measured during the discrimination task will generally reflect contributions of both the sensory stimulus and the perceptual decision (Zaidel et al., 2017), we analyzed the relationships between neural responses, heading, and choice using a partial correlation analysis (Zaidel et al., 2017). Response PSTHs of an example PIVC neuron are shown in Figure $5 \mathrm{~A}$. Tuning curves calculated during the acceleration and deceleration time windows are shown in Figure 5B,C, black symbols and lines, respectively. To decouple the effects of stimulus and choice on neural activity, we also computed heading tuning curves conditioned on the animal's choices (Fig. 5B,C, cyan and magenta curves). For headings near zero, where the animal makes many choices in both directions, choice-related activity can be assessed by looking at the difference in response between rightward $(\boldsymbol{)})$ and leftward (4) choices for the same heading value on the abscissa. This example neuron shows a clear choice effect during the acceleration window, with greater responses for leftward choices. Note, however, that this neuron's stimulus preference is for rightward (positive) headings, such that effects of stimulus and choice on neural responses have opposite polarities.

To summarize and quantify the contributions of heading and choice to neuronal responses, we performed a partial correlation analysis (see Materials and Methods; Zaidel et al., 2017). In this analysis, positive (negative) heading partial correlations reflect an increase in firing rate for rightward (leftward) headings, while positive (negative) choice partial correlations reflect an increase in firing rate for rightward (leftward) choices. For the example neuron in Figure 5, the partial correlation analysis reveals significant contributions of both heading and choice for the acceleration window $\left(r_{\text {heading }}=0.55, p=1.0 \times 10^{-30}, r_{\text {choice }}=-0.31\right.$, $\left.p=4.2 \times 10^{-13}\right)$ and the deceleration window $\left(r_{\text {heading }}=0.61\right.$, $\left.p=1.0 \times 10^{-30}, r_{\text {choice }}=-0.20, p=3.8 \times 10^{-6}\right)$. Note that the heading and choice partial correlations have opposite signs in both time windows.

Results of the partial correlation analysis for the population of PIVC neurons are shown in Figure $6 A-C$. For a more complete comparison with MSTd and VIP datasets, we computed partial correlations within a time window centered around peak stimulus velocity, in addition to the acceleration and deceleration windows. For the acceleration window (Fig. 6A), both heading and choice contribute to PIVC responses, with $40.7 \%$ (44/108) of neurons having a significant heading partial correlation, $27.8 \%$ $(30 / 108)$ of neurons having a significant choice partial correlation, and $17.6 \%(19 / 108)$ of neurons having significant partial 
correlations for both heading and choice. To compare the contributions of heading and choice to PIVC responses, we applied Fisher's $r$ to $z$-transformation to these partial correlation coefficients (Levy and Narula, 1978) and compared the magnitudes of the $\mathrm{z}$ values. The heading partial correlation, as measured by the mean value of $|z|(0.134 \pm 0.012 \mathrm{SE})$, is significantly greater than the corresponding mean $|z|$ value $(0.099 \pm 0.010 \mathrm{SE})$ for the choice partial correlation $(p=0.0179$, Wilcoxon rank test). This indicates that the influence of stimulus heading is stronger than the influence of choice during the acceleration time window.

Also, there is a significant negative slope between heading and choice partial correlations in the acceleration window $\left(r=-0.41, p=9.9 \times 10^{-6}\right.$, Pearson correlation, $N=108, \mathrm{df}=106$ ), which indicates that heading and choice contributions to PIVC responses are generally mismatched in polarity. To examine whether this pattern is related to the acceleration dynamics of neural responses, we examined this relationship separately for SP and DP cells. Interestingly, we find a strong negative correlation between heading and choice signals for DP cells $(r=$ $-0.605, p=6.2 \times 10^{-7}$, Pearson's correlation, $N=57$, $\mathrm{df}=55$ ) but not for SP cells $(r=-0.085, p=0.551$, Pearson's correlation, $N=51, \mathrm{df}=49$ ). Accordingly, there is a significant difference in the relationship of choice to heading partial correlations between these two groups of cells in the acceleration window $(\mathrm{z}=-3.10, p=0.0019$, $\mathrm{Z}$ test).

For the peak velocity (Fig. 6B) and deceleration (Fig. 6C) time windows, stimulus heading and choice made roughly similar contributions to PIVC responses. For the velocity window, $45.4 \% \quad(49 / 108), \quad 28.7 \% \quad(31 / 108)$, and $16.7 \%(18 / 108)$ of neurons have significant heading, choice, and both partial correlations, respectively; for the deceleration window, $47.2 \%$ (51/108), 32.4\% $(35 / 108)$, and $15.7 \%(17 / 108)$ of neurons have significant heading, choice and both partial correlations, respectively. Again, we found a tendency for choice partial correlations to be anti-correlated with heading partial correlations for the entire population of PIVC neurons (velocity window: $r=$ $-0.25, p=0.008$; deceleration window: $r=$

$-0.13, p=0.1859$, Pearson's correlation, $N=108, \mathrm{df}=106)$. These negative correlations, particularly in the velocity window, again appear to be mainly driven by DP cells (velocity window: $r=-0.40$, $p=0.002$; deceleration window: $r=-0.1151, p=0.3939$, Pearson's correlation, $N=57, \mathrm{df}=55$ ), not SP cells (velocity window: $r=0.15$; $p=0.27$; deceleration window: $r=-0.1595, p=0.2637$, Pearson's
A

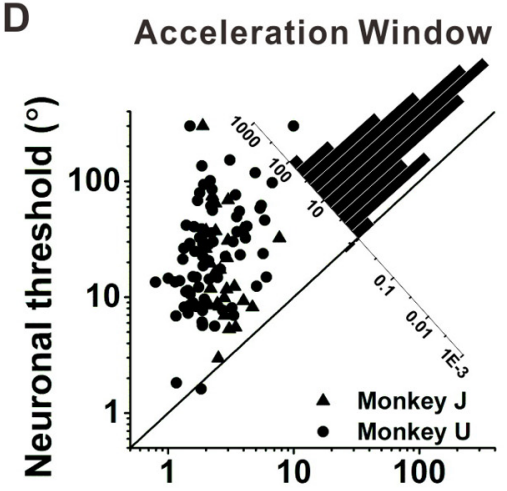

Behavioral threshold $\left({ }^{\circ}\right)$
옴
B

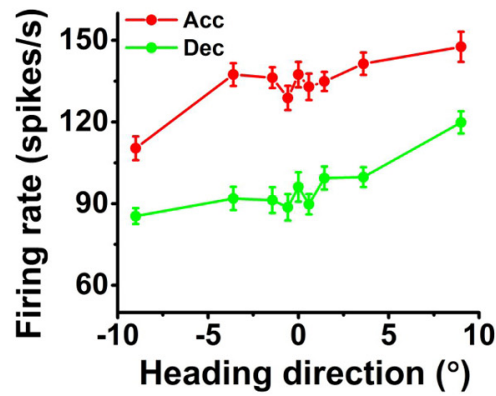

C

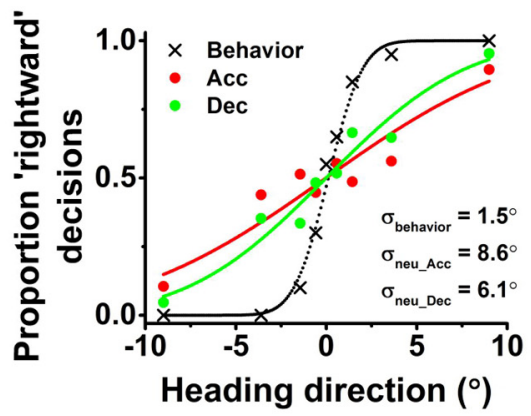

Figure 4. Quantification of neuronal sensitivity in area PIVC. A, Average PSTHs for each of nine headings tested in the discrimination task. Each PSTH was constructed using 25-ms time bins and was smoothed with a 400-ms boxcar filter. Vertical dashed red and green lines indicate the times of peak stimulus acceleration and deceleration ( $t=0.82$ and $1.18 \mathrm{~s}$ ). Stimulus velocity (dashed line), acceleration (black solid line), and position (dotted line) profiles are overlaid for comparison (bottom row). $\boldsymbol{B}$, Firing rates (mean $\pm \mathrm{SE}$ ) of the same cell for the two time windows (red, acceleration window; green, deceleration window), plotted as a function of heading (positive values indicate rightward headings). C, Psychometric function (black $\mathrm{x}$ ) and neurometric functions corresponding to the acceleration (red, $0.82 \mathrm{~s}$ ) and deceleration (green, $1.18 \mathrm{~s}$ ) time windows. Smooth curves show cumulative Gaussian fits. Threshold values that summarize behavioral $\left(\sigma_{\text {behavior }}\right)$ and neuronal $\left(\sigma_{\text {neu_Acc, }} \sigma_{\text {neu_Dec }}\right)$ performance are given. $\boldsymbol{D}, \boldsymbol{E}$, Summary of the relationship between neuronal and psychophysical thresholds for the acceleration $(\boldsymbol{D})$ and deceleration $(\boldsymbol{E})$ time windows. Diagonal marginal histograms indicate the distributions of the ratio of neuronal:psychophysical thresholds.

correlation, $N=51, \mathrm{df}=49$ ). The difference in correlation between the groups was significant for the velocity window $(z=2.898$, $p=0.0038)$ but not for the deceleration window $(\mathrm{z}=-0.228$, $p=0.8195)$.

For DP cells, stimulus heading tended to make a greater contribution than choice to PIVC responses in the velocity and deceleration windows, as indicated by the greater horizontal 
A
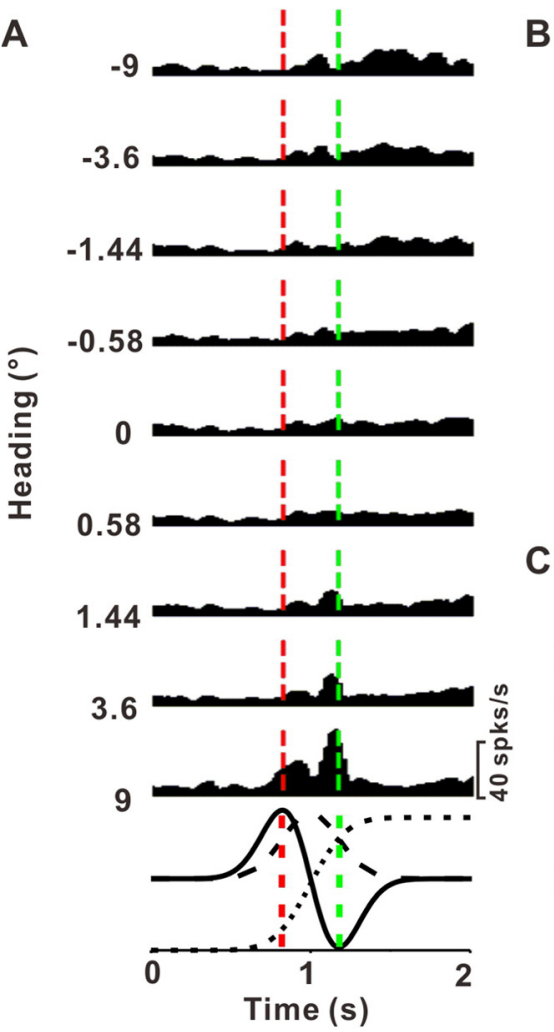

B

\section{Acceleration Window}

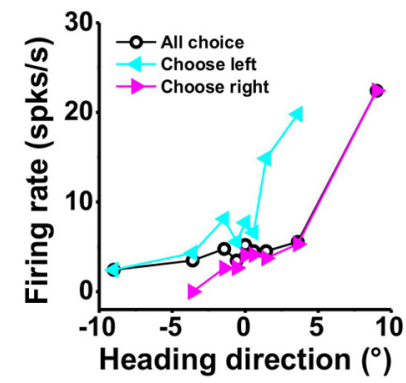

C

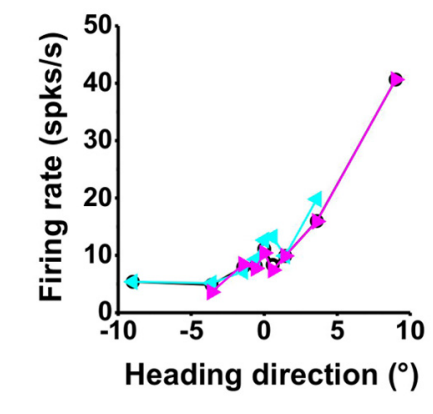

Figure 5. Computation of choice-conditioned tuning curves for an example PIVC neuron. $\boldsymbol{A}$, Average PSTHs for the nine headings tested in the discrimination task, with 400-ms analysis window slid across the data in steps of $25 \mathrm{~ms}$. Red and green lines indicate the times of peak stimulus acceleration and deceleration $(t=0.82$ and 1.18 s). Format as in Figure $4 A$. $\boldsymbol{B}$, Responses of the same cell for the acceleration window, plotted as a function of heading. The black curve indicates the heading tuning based on all trials. Cyan and magenta curves show choiceconditioned heading tuning curves corresponding to leftward and rightward choices, respectively. $\boldsymbol{C}$, Heading tuning curves for the deceleration time window. Format as in Figure $4 B$.

spread of the data (Fig. 6B,C, pink). Correspondingly, the magnitude of heading partial correlations, as measured by the mean value of $|z|$ (velocity window: 0.1635; deceleration window: 0.1823 ), was greater than that of choice partial correlations (velocity window: 0.1237; deceleration window: 0.1359$)$ for the velocity and deceleration windows $(p=0.0259$ and $p=0.0442$, respectively, Wilcoxon rank test, $N=57)$. In contrast, for SP cells, we found no significant difference between the mean values of $\mathrm{z} \mid$ for heading and choice $(0.0940$ and 0.0934 , respectively; $p=$ 0.8374 , Wilcoxon rank test, $N=51$ ) in the velocity window, nor between the corresponding mean values of $|z|$ (0.1101 and $0.0926)$ for heading and choice in the deceleration window ( $p=0.2232$, Wilcoxon rank test, $N=51$ ). For all three time windows, we found no significant differences between animals in the mean absolute values of the $\mathrm{z}$-transformed partial correlation values (acceleration window: $p=0.095$ for heading and 0.1638 for choice; velocity window: $p=0.1214$ for heading and 0.4907 for choice; deceleration window: $p=0.3659$ for heading and 0.7445 for choice, respectively, two-way ANOVA).

Similar to PIVC, MSTd shows a dominance of heading over choice signals in all three time windows (Fig. $6 D-F$ ), with the magnitude (mean $|z|$ value) of heading partial correlations (acceleration: 0.11125 ; velocity: 0.12079; deceleration: 0.12972 ) being significantly greater than the magnitude of choice partial correlations (acceleration: $0.06758, p=7.8 \times 10^{-5}$; velocity: $0.07607, p=$ 0.0038; deceleration: $0.07584, p=3.9 \times 10^{-5}$; Wilcoxon rank test, $N=182$ ). In contrast to PIVC, MSTd neurons show a positive slope to the relationship between heading and choice partial correlations (acceleration window: $r=0.2386, p=0.0012$; velocity window: $r=0.2033, p=0.0059$; deceleration window: $r=0.1590, p=0.0320$, Pearson correlation, $N=182 ; \mathrm{df}=180$ ), indicating that stimulus and choice generally have similar influences on neural responses, as one would expect in a feedforward system, for example (Zaidel et al., 2017). For all three time windows, this positive correlation appears to be driven more strongly by SP cells (acceleration: $r=0.2982, p=6.96 \times 10^{-4}$; velocity: $r=0.2870, p=0.0011$; deceleration: $r=0.2490$, $p=0.0049$, Pearson's correlation, $N=126$, $\mathrm{df}=124$ ) than DP cells (acceleration: $r=0.1414$, $p=0.2985$; velocity: $r=0.1117, p=0.4126$; deceleration: $r=0.0339, p=0.8039$, Pearson's correlation, $N=56, \mathrm{df}=54$ ), although the larger sample of SP cells might account for the significant correlation in that case. Indeed, the difference in slope between the two groups of cells was not significant (acceleration window: $\mathrm{z}=1.005, p=0.3147$ velocity window: $\mathrm{Z}=1.115, \quad p=0.2651 ; \quad$ deceleration window: $\mathrm{Z}=1.342, p=0.1797, \mathrm{Z}$ test).

For VIP, choice effects on neural responses are dominant, with the magnitude (mean $|z|$ value) of heading partial correlations (acceleration: 0.10188; velocity: 0.10614 ; deceleration: $0.12612, N=68$ ) being significantly less than the magnitude of choice partial correlations (acceleration: 0.17962; velocity: 0.19371; deceleration: $0.17864, N=68$ ) in all three time windows $\left(p=9.5 \times 10^{-4}, p=0.0011\right.$ and $p=0.0254$, respectively, Wilcoxon rank test, $N=68$ ). For all three time windows, there are no significant correlations to the relationship between heading and choice partial correlations for VIP (acceleration: $r=0.0470, p=0.7036$; velocity: $r=$ -0.1804, $p=0.1411$; deceleration: $r=0.1246, p=0.3112$, Pearson correlation, $N=68, \mathrm{df}=66$ ). And there is no significant difference in slope between SP and DP cells (acceleration window: $z=0.088$, $p=0.9298$; velocity window: $\mathrm{z}=1.256, p=0.2090$; deceleration window: $\mathrm{z}=-1.791, p=0.0733, \mathrm{Z}$ test).

Overall, the data of Figure 6 reveal a different balance of heading and choice signals across areas, as well as different signs of the relationship between heading and choice partial correlations (see Discussion).

\section{Time course of heading and choice signals in PIVC versus MSTd and VIP}

Since the motion stimuli were dynamic, with a Gaussian velocity profile and a biphasic acceleration profile (Fig. 1C), we next examined the time course of heading and choice partial correlations using 25-ms time bins smoothed with a 100-ms moving window, as shown in Figure 7. To avoid positive and negative partial correlations cancelling when we take the average across neurons at each time point (see also Zaidel et al., 2017), we plotted the time course of the average of the squared partial correlations (denoted by $R^{2}$ ). For the time course of squared heading partial correlation, we used ARIMA(p,d,q) models of order $(0,1$, 2 ) for SP neurons and $(3,1,2)$ for DP neurons, which means that there are some linear trends in the time series of responses for SP and DP neurons, and we first need one time differencing step $(d=1)$ to make the time series stationary, then two prior errors 


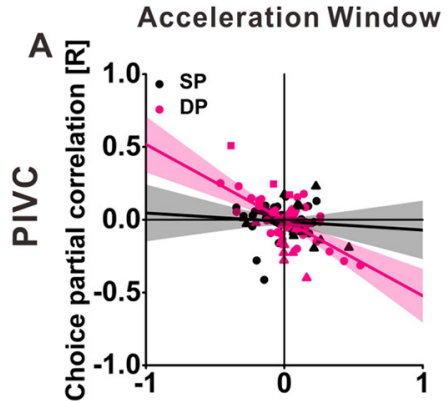

Heading partial correlation [R]

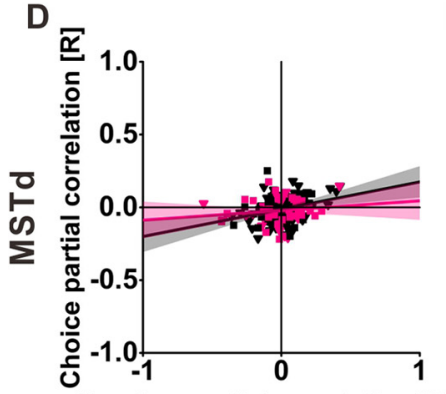

Heading partial correlation [R]

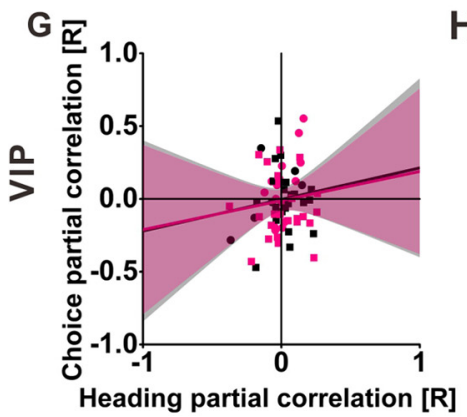

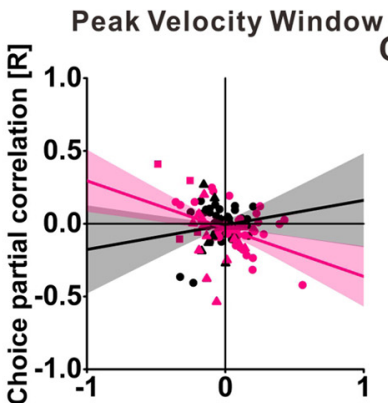

Heading partial correlation [R]
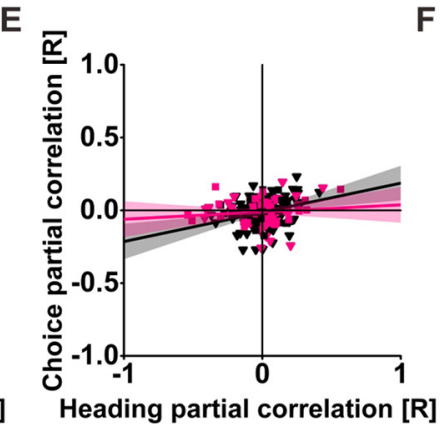

$\mathrm{H}$

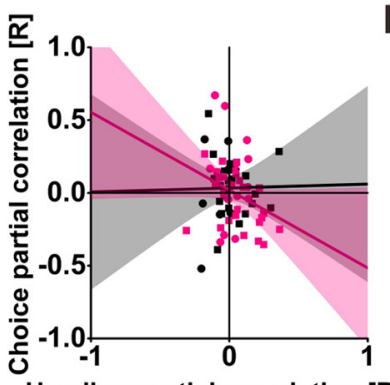

Heading partial correlation $[R]$

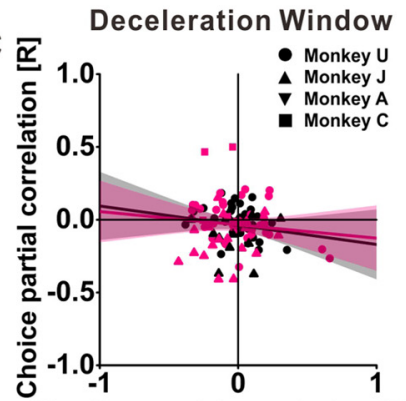

$\mathbf{F}$

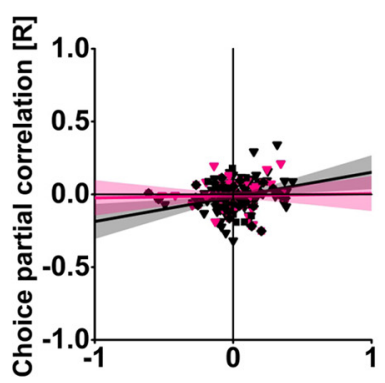

Heading partial correlation [R]

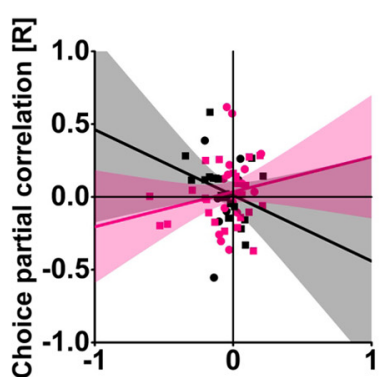

Heading partial correlation [R]

Figure 6. Relationships between choice and heading partial correlations for areas PIVC $(\boldsymbol{A}-\boldsymbol{C})$, MSTd $(\boldsymbol{D}-\boldsymbol{F})$, and VIP $(\boldsymbol{G}-\boldsymbol{I})$ during acceleration $(\boldsymbol{A}, \boldsymbol{D}, \boldsymbol{G})$, peak velocity $(\boldsymbol{B}, \boldsymbol{E}, \boldsymbol{H})$, and deceleration $(C, F, I)$ time windows. Partial correlations were calculated between neuronal firing rates and stimulus headings (given the actual choices made by the monkey) or choices (given the stimulus heading). Each data point represents choice and heading partial correlation coefficients calculated using a 100-ms analysis window centered at peak acceleration $(t=0.82 \mathrm{~s})$, velocity $(t=$ $1.0 \mathrm{~s})$, and deceleration $(t=1.18 \mathrm{~s})$ of the motion profile for one cell. Data are shown separately for SP cells (black symbols) and DP cells (pink symbols). Solid lines and the shaded regions represent Type-II regressions with their $95 \%$ confidence intervals.

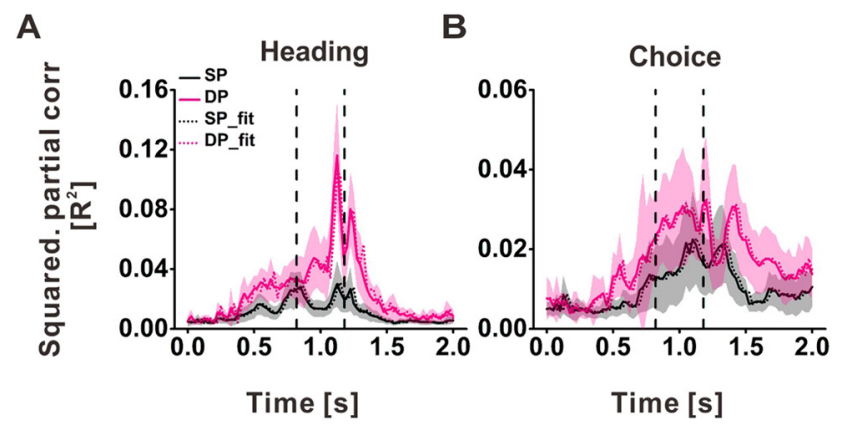

Figure 7. Time course heading and choice signals in PIVC. $\boldsymbol{A}, \boldsymbol{B}$, Temporal profiles of squared partial correlation coefficients $\left(R^{2}\right)$ for heading $(\boldsymbol{A})$ and choice $(\boldsymbol{B})$, plotted separated for SP (solid black) and DP (solid pink) cells. Error bands represent 95\% confidence intervals. The dashed lines show the corresponding ARIMA fits.

for SP neurons, and three prior values of the series together with the two prior errors in estimation for DP neurons to get the best estimate of each value in the series (for details, see Materials and Methods). ARIMA models capture the variation in each time series explained by its own prior history, the MA model uses the errors from the past forecasts to predict the future values, while the AR model uses the past forecasts to predict future values. The different parameters of ARIMA fits between SP and DP neurons suggest that these two groups of neurons respond to heading stimuli with different dynamics. Accordingly, the time course of squared heading partial correlation for DP cells has a substantially different structure from that of SP cells $\left(F_{(1,160)}=\right.$ 15.11, $p=9.9 \times 10^{-7}$ for SP vs DP, chow test; Fig. $\left.7 A\right)$.

For the time courses of choice-related activity (Fig. $7 B$ ), we used ARIMA models of order $(0,1,1)$ for SP neurons and $(0,1$, 2) for DP neurons. In this case, the time course of squared partial correlation for DP cells was also significantly different from that of single peaked cells $\left(F_{(1,160)}=25.45, p=2.6 \times 10^{-10}\right.$ for SP vs DP, chow test). The difference across animals for the time course of the squared heading partial correlation was not significant $\left(F_{(1,160)}=1.4730, p=0.2324\right.$; chow test $)$, although the time course of squared choice partial correlation across animals was significantly different $\left(F_{(1,40)}=30.2308, p=7.6 \times 10^{-12}\right.$; chow test). Overall, these results suggest that the temporal response profiles of SP and DP neurons are different and they might use different strategies to process heading and choice-related signals during self-motion perception. 
A

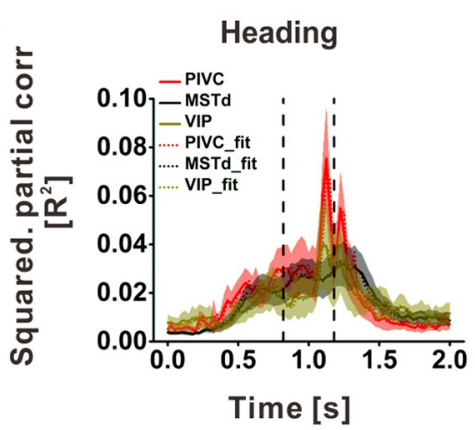

B

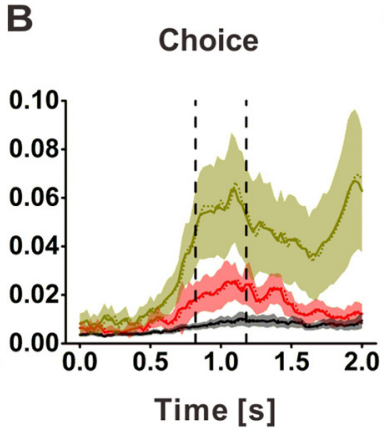

C

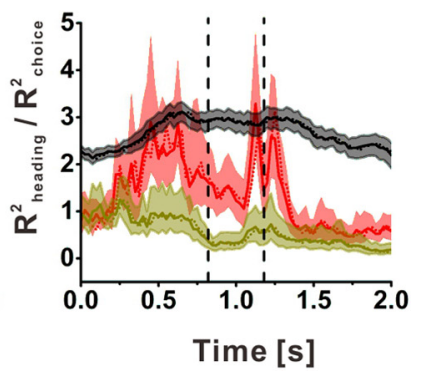

Figure 8. Comparison of time course of heading and choice signals between areas PIVC, MSTd, and VIP. $A$, Time course of the average squared heading partial correlation for PIVC (red), MSTd (black), and VIP (dark yellow). Error bands represent $95 \%$ confidence intervals. B, Time courses of the average squared choice partial correlations. Format as in panel $\boldsymbol{A}$. C, The ratio of the mean for squared heading versus choice partial correlations across time in PIVC, MSTd, and VIP. The shaded areas correspond to 95\% confidence intervals obtained using the bootstrap analysis. Dashed lines in each panel show ARIMA fits to the data.

We further compared the temporal dynamics of heading and choice signals among PIVC, MSTd, and VIP, as shown in Figure 8. For the time course of heading signals, the ARIMA models for PIVC, VIP, and MSTd had parameters given by $(3,1,2),(5,2$, $2)$, and $(2,1,3)$, respectively. The squared heading partial correlation in PIVC had a similar temporal structure with MSTd and VIP (PIVC vs MSTd: $F_{(1,160)}=1.8081, p=0.1673$; PIVC vs VIP: $F_{(1,160)}=1.7095, p=0.1843$; MSTd vs VIP: $F_{(1,160)}=0.2370, p=$ 0.7893 , chow test). This indicates that the dynamics of headingrelated signals among these three areas are broadly similar, although the maximum squared heading partial correlation is greater in PIVC than MSTd and VIP around the time of peak deceleration. In contrast, the time courses of squared partial correlations for choice (Fig. $8 B$ ) differ greatly among areas throughout the stimulus period $\left(F_{(1,160)}=55.3057, p=6.2 \times 10^{-19}\right.$ for PIVC vs MSTd; $F_{(1,160)}=157.0214, p=2.8 \times 10^{-38}$ for PIVC vs VIP; $F_{(1,160)}=304.8759, p=5.8 \times 10^{-55}$ for MSTd vs VIP, chow test), as revealed by fitting the ARIMA model with parameters of $(0,1,1),(4,1,2)$, and $(2,2,2)$ for PIVC, VIP, and MSTd, respectively. To summarize these patterns, we plotted the ratio of the mean $R^{2}$ values for heading and choice as a function of time for each area (Fig. $8 C$ ). The ratios for PIVC have an overall magnitude that lies in between MSTd and VIP, indicating more balanced contributions from stimulus and choice signals in PIVC.

\section{Discussion}

In the last few decades, research has made great strides toward explaining how the brain computes accurate estimates of our self-motion relative to the world (Britton and Arshad, 2019). In particular, the vestibular system makes important contributions to our perception of self-motion, and various cortical regions in primates have been characterized with regard to vestibular heading selectivity (Gu et al., 2006, 2007; Chen et al., 2010, 2011a,b, 2013). Among these areas, inactivation of the PIVC substantially impaired the precision of vestibular heading discrimination (Chen et al., 2016) while large bilateral injections of muscimol in VIP or MSTd reveal no deficits or very weak deficits in performance, respectively (Chen et al., 2016).

Theories of perceptual decision-making have considered that decision-related neural activity in sensory cortex can be derived from bottom-up (feedforward) impact of sensory propagation of noise (Britten et al., 1996; Shadlen et al., 1996; Kanitscheider et al., 2015; Pitkow et al., 2015). In a feedforward model, sensory neurons undergo collective noise fluctuations that influence the outcome of the decision process and hence create a correlation between neuronal and behavioral responses. Responses of individual task-relevant sensory neurons can therefore predict monkeys' trial-by-trial choices in perceptual decision-making tasks. Thus, choice-correlated activity has sometimes been interpreted as evidence that the responses of these neurons are causally linked to perceptual judgments (Britten et al., 1996; Shadlen et al., 1996; Kanitscheider et al., 2015; Pitkow et al., 2015). However, there has been growing evidence that contradicts this simple feed-forward hypothesis and instead emphasizes the role of top-down (feedback) signals in the origin of choice-related activity, in part by showing that the correlated noise in populations of sensory neurons changes with attentional state or other task demands (Cohen and Newsome, 2008; Cohen and Maunsell, 2009; Mitchell et al., 2009; Nienborg and Cumming, 2009; Ruff and Cohen, 2014; Yang et al., 2016; Bondy et al., 2018).

If choice signals in PIVC primarily reflected a feedforward, causal contribution of sensory neurons to decisions, one would have expected greater choice-related signals in PIVC during vestibular self-motion perception than the other areas. However, we found that choice signals were balanced with heading signals in PIVC, and were significantly weaker than choice signals in VIP, although greater than in MSTd. Thus, our present findings support previous conclusions that the interpretation of choice signals is complex (Zaidel et al., 2017) and does not just imply feedforward causality (Nienborg and Cumming, 2009). Our findings also agree with the relationship between microstimulation effects and choice-correlations observed by $\mathrm{Yu}$ and $\mathrm{Gu}$ (2018) in several visual/visualmotor areas (MT, MST, and VIP) during motion discrimination. In fact, the activity of sensory neurons is modulated by a variety of contextual factors, such as adaptation, attention, wakefulness, reward, and locomotion (Luck et al., 1997; Kato et al., 2012; Benucci et al., 2013; Aydın et al., 2018; Le Merre et al., 2018; Guo et al., 2019), and perceptual decisions are influenced by such factors as recent stimulus, choice, and feedback history (Lau and Glimcher, 2005; Fischer and Whitney, 2014; Abrahamyan et al., 2016). These phenomena might conspire to create correlations between neuronal and behavioral responses. Additionally, some computational studies have demonstrated that choice-correlated activities in task-relevant neurons arise from an inference process involving both feedforward and feedback computations (Wimmer et al., 2015; Haefner et al., 2016).

One of the most salient findings of the present study is that choice signals are negatively correlated with sensory signals in DP cells. This finding appears contradictory to the feedforward interpretation of choice signals, which predicts that stimulus- 
related and choice-related modulations should have the same sign, as indicated by the positive correlation between heading versus choice signals as observed in MSTd (Fig. 6D-F). One possibility is that the negative correlation may be caused by the acceleration dynamics in DP cells. Given the biphasic nature of acceleration dynamics, there are essentially opposite preferred directions at different times for DP cells. Perhaps the negative correlation between choice and heading partial correlations in the early window reflects how DP cells are decoded. We also saw some weak negative correlation in deceleration window, but we cannot rule out the sequential effects of adaptation since the acceleration was always followed by deceleration. One interpretation of the strong PIVC inactivation results is its consideration as the closest to a "vestibular cortex" (Guldin and Grüsser, 1998; Brandt and Dieterich, 1999; Chen et al., 2010). Indeed, PIVC is closer than MSTd and VIP to the peripheral vestibular system, receiving direct vestibular projections from the thalamus (Chen et al., 2011c). Thus, inactivation of PIVC may serve to block some of the bottom-up propagation of sensory information, which would severely impair heading discrimination performance.

Returning to the top-down modulation framework, previous studies have suggested some additional hypotheses, e.g., the sharpening hypothesis and the prediction error hypothesis. The sharpening hypothesis states that top-down signals enhance the neural representation in the lower sensory areas, thus improving the quality of the degraded sensory signal (Hsieh et al., 2010; Abdelhack and Kamitani, 2018). Indeed, several studies have shown evidence for sharpened signals driven by prediction in the lower visual areas (Lee and Mumford, 2003; Hsieh et al., 2010; Kok et al., 2012; Gayet et al., 2017). But this scheme would seem to also predict a positive correlation between stimulus and choice partial correlations, which were not observed in PIVC. Conversely, the prediction error hypothesis states that top-down signals provide expected signal information that would be redundant if represented again in lower sensory areas, and therefore gets subtracted (Mumford, 1992; Rao and Ballard, 1999). This results in an error signal that is repeatedly processed to update the prediction signal until the error signal reaches zero, which corresponds with achieving a perceptual result (Murray et al., 2002; den Ouden et al., 2009; Alink et al., 2010; Meyer and Olson, 2011; Todorovic et al., 2011; Kok et al., 2012; Gordon et al., 2017). Such a framework might be compatible with the negative correlation between sensory and choice signals observed in PIVC. Since the negative slope between heading and choice partial correlations was produced mostly by DP cells, the top-down suppression might be related with spatiotemporal acceleration signals in PIVC. For example, the acceleration signals might play a role of a prediction error, similar to the bidirection modulation observed in the dopaminergic system (Schultz et al., 1997). These hypotheses remain to be tested in future experiments.

\section{References}

Abdelhack M, Kamitani Y (2018) Sharpening of hierarchical visual feature representations of blurred images. eNeuro 5:ENEURO.0443-17.2018.

Abrahamyan A, Silva LL, Dakin SC, Carandini M, Gardner JL (2016) Adaptable history biases in human perceptual decisions. Proc Natl Acad Sci USA 113:E3548-E3557.

Alink A, Schwiedrzik CM, Kohler A, Singer W, Muckli L (2010) Stimulus predictability reduces responses in primary visual cortex. J Neurosci 30:2960-2966.

Aydın Ç, Couto J, Giugliano M, Farrow K, Bonin V (2018) Locomotion modulates specific functional cell types in the mouse visual thalamus. Nat Commun 9:4882.
Benucci A, Saleem AB, Carandini M (2013) Adaptation maintains population homeostasis in primary visual cortex. Nat Neurosci 16:724-729.

Bondy AG, Haefner RM, Cumming BG (2018) Feedback determines the structure of correlated variability in primary visual cortex. Nat Neurosci 21:598-606.

Brandt T, Dieterich M (1999) The vestibular cortex. Its locations, functions, and disorders. Ann NY Acad Sci 871:293-312.

Britten KH, Newsome WT, Shadlen MN, Celebrini S, Movshon JA (1996) A relationship between behavioral choice and the visual responses of neurons in macaque MT. Vis Neurosci 13:87-100.

Britton Z, Arshad Q (2019) Vestibular and multi-sensory influences upon self-motion perception and the consequences for human behavior. Front Neurol 10:63.

Chen A, DeAngelis GC, Angelaki DE (2010) Macaque parieto-insular vestibular cortex: responses to self-motion and optic flow. J Neurosci 30:30223042.

Chen A, DeAngelis GC, Angelaki DE (2011a) Representation of vestibular and visual cues to self-motion in ventral intraparietal cortex. J Neurosci 31:12036-12052.

Chen A, DeAngelis GC, Angelaki DE (2011b) Convergence of vestibular and visual self-motion signals in an area of the posterior sylvian fissure. J Neurosci 31:11617-11627.

Chen A, DeAngelis GC, Angelaki DE (2011c) A comparison of vestibular spatiotemporal tuning in macaque parietoinsular vestibular cortex, ventral intraparietal area, and medial superior temporal area. J Neurosci 31:3082-3094

Chen A, DeAngelis GC, Angelaki DE (2013) Functional specializations of the ventral intraparietal area for multisensory heading discrimination. J Neurosci 33:3567-3581.

Chen A, Gu Y, Liu S, DeAngelis GC, Angelaki DE (2016) Evidence for a causal contribution of macaque vestibular, but not intraparietal, cortex to heading perception. J Neurosci 36:3789-3798.

Cohen MR, Maunsell JH (2009) Attention improves performance primarily by reducing interneuronal correlations. Nat Neurosci 12:1594-1600.

Cohen MR, Newsome WT (2008) Context-dependent changes in functional circuitry in visual area MT. Neuron 60:162-173.

Cumming BG, Nienborg H (2016) Feedforward and feedback sources of choice probability in neural population responses. Curr Opin Neurobiol 37:126-132.

den Ouden HE, Friston KJ, Daw ND, McIntosh AR, Stephan KE (2009) A dual role for prediction error in associative learning. Cereb Cortex 19:1175-1185

Fetsch CR, DeAngelis GC, Angelaki DE (2010) Visual-vestibular cue integration for heading perception: applications of optimal cue integration theory. Eur J Neurosci 31:1721-1729.

Fischer J, Whitney D (2014) Serial dependence in visual perception. Nat Neurosci 17:738-743.

Gayet S, Guggenmos M, Christophel TB, Haynes JD, Paffen CLE, Van der Stigchel S, Sterzer P (2017) Visual working memory enhances the neural response to matching visual input. J Neurosci 37:6638-6647.

Gordon N, Koenig-Robert R, Tsuchiya N, van Boxtel JJ, Hohwy J (2017) Neural markers of predictive coding under perceptual uncertainty revealed with hierarchical frequency tagging. Elife 6:e22749.

Goris RLT, Ziemba CM, Stine GM, Simoncelli EP, Movshon JA (2017) Dissociation of choice formation and choice-correlated activity in macaque visual cortex. J Neurosci 37:5195-5203.

Gu Y, Watkins PV, Angelaki DE, DeAngelis GC (2006) Visual and nonvisual contributions to three-dimensional heading selectivity in the medial superior temporal area. J Neurosci 26:73-85.

Gu Y, DeAngelis GC, Angelaki DE (2007) A functional link between area MSTd and heading perception based on vestibular signals. Nat Neurosci 10:1038-1047.

Gu Y, Angelaki DE, DeAngelis GC (2008) Neural correlates of multisensory cue integration in macaque MSTd. Nat Neurosci 11:1201-1210.

Gu Y, DeAngelis GC, Angelaki DE (2012) Causal links between dorsal medial superior temporal area neurons and multisensory heading perception. J Neurosci 32:2299-2313.

Guldin WO, Grüsser OJ (1998) Is there a vestibular cortex? Trends Neurosci 21:254-259.

Guo L, Weems JT, Walker WI, Levichev A, Jaramillo S (2019) Choice-selective neurons in the auditory cortex and in its striatal target encode reward expectation. J Neurosci 39:3687-3697. 
Haefner RM, Berkes P, Fiser J (2016) Perceptual decision-making as probabilistic inference by neural sampling. Neuron 90:649-660.

Harris LR, Jenkin M, Zikovitz DC (2000) Visual and non-visual cues in the perception of linear self-motion. Exp Brain Res 135:12-21.

Hsieh PJ, Vul E, Kanwisher N (2010) Recognition alters the spatial pattern of FMRI activation in early retinotopic cortex. J Neurophysiol 103:15011507.

Kanitscheider I, Coen-Cagli R, Pouget A (2015) Origin of information-limiting noise correlations. Proc Natl Acad Sci USA 112:E6973-E6982.

Kato HK, Chu MW, Isaacson JS, Komiyama T (2012) Dynamic sensory representations in the olfactory bulb: modulation by wakefulness and experience. Neuron 76:962-975.

Kok P, Jehee JF, de Lange FP (2012) Less is more: expectation sharpens representations in the primary visual cortex. Neuron 75:265-270.

Lau B, Glimcher PW (2005) Dynamic response-by-response models of matching behavior in rhesus monkeys. J Exp Anal Behav 84:555-579.

Le Merre P, Esmaeili V, Charrière E, Galan K, Salin PA, Petersen CCH, Crochet S (2018) Reward-based learning drives rapid sensory signals in medial prefrontal cortex and dorsal hippocampus necessary for goaldirected behavior. Neuron 97:83-91.e5.

Lee TS, Mumford D (2003) Hierarchical Bayesian inference in the visual cortex. J Opt Soc Am A Opt Image Sci Vis 20:1434-1448.

Levy KJ, Narula SC (1978) Testing hypotheses concerning partial correlations - some methods and discussion. Int Stat Rev 46:215-218.

Luck SJ, Chelazzi L, Hillyard SA, Desimone R (1997) Neural mechanisms of spatial selective attention in areas V1, V2, and V4 of macaque visual cortex. J Neurophysiol 77:24-42.

McNaughton BL, Battaglia FP, Jensen O, Moser EI, Moser MB (2006) Path integration and the neural basis of the 'cognitive map'. Nat Rev Neurosci 7:663-678.

Meyer T, Olson CR (2011) Statistical learning of visual transitions in monkey inferotemporal cortex. Proc Natl Acad Sci USA 108:19401-19406.

Mitchell JF, Sundberg KA, Reynolds JH (2009) Spatial attention decorrelates intrinsic activity fluctuations in macaque area V4. Neuron 63:879-888.

Mumford D (1992) On the computational architecture of the neocortex. II. The role of cortico-cortical loops. Biol Cybern 66:241-251.

Murray SO, Kersten D, Olshausen BA, Schrater P, Woods DL (2002) Shape perception reduces activity in human primary visual cortex. Proc Natl Acad Sci USA 99:15164-15169.

Newbold P (1983) Arima model-building and the time-series analysis approach to forecasting. J Forecast 2:23-35.
Nienborg H, Cumming BG (2009) Decision-related activity in sensory neurons reflects more than a neuron's causal effect. Nature 459:89-92.

Ohmi M (1996) Egocentric perception through interaction among many sensory systems. Brain Res Cogn Brain Res 5:87-96.

Pitkow X, Liu S, Angelaki DE, DeAngelis GC, Pouget A (2015) How can single sensory neurons predict behavior? Neuron 87:411-423.

Prokhorov AV (2001) Partial correlation coefficient (Hazewinkel M, ed). Encyclopedia of Mathematics, Springer.

Rao RP, Ballard DH (1999) Predictive coding in the visual cortex: a functional interpretation of some extra-classical receptive-field effects. Nat Neurosci 2:79-87.

Ruff DA, Cohen MR (2014) Attention can either increase or decrease spike count correlations in visual cortex. Nat Neurosci 17:1591-1597.

Schultz W, Dayan P, Montague PR (1997) A neural substrate of prediction and reward. Science 275:1593-1599.

Shadlen MN, Britten KH, Newsome WT, Movshon JA (1996) A computational analysis of the relationship between neuronal and behavioral responses to visual motion. J Neurosci 16:1486-1510.

Stackman RW, Clark AS, Taube JS (2002) Hippocampal spatial representations require vestibular input. Hippocampus 12:291-303.

Takahashi K, Gu Y, May PJ, Newlands SD, DeAngelis GC, Angelaki DE (2007) Multimodal coding of three-dimensional rotation and translation in area MSTd: comparison of visual and vestibular selectivity. J Neurosci 27:9742-9756.

Telford L, Howard IP, Ohmi M (1995) Heading judgments during active and passive self-motion. Exp Brain Res 104:502-510.

Todorovic A, van Ede F, Maris E, de Lange FP (2011) Prior expectation mediates neural adaptation to repeated sounds in the auditory cortex: an MEG study. J Neurosci 31:9118-9123.

Wimmer K, Compte A, Roxin A, Peixoto D, Renart A, de la Rocha J (2015) Sensory integration dynamics in a hierarchical network explains choice probabilities in cortical area MT. Nat Commun 6:6177.

Yang H, Kwon SE, Severson KS, O'Connor DH (2016) Origins of choicerelated activity in mouse somatosensory cortex. Nat Neurosci 19:127134.

Yoder RM, Taube JS (2014) The vestibular contribution to the head direction signal and navigation. Front Integr Neurosci 8:32.

Yu X, Gu Y (2018) Probing sensory readout via combined choice-correlation measures and microstimulation perturbation. Neuron 100:715-727.e5.

Zaidel A, DeAngelis GC, Angelaki DE (2017) Decoupled choice-driven and stimulus-related activity in parietal neurons may be misrepresented by choice probabilities. Nat Commun 8:715. 